\title{
Spin-Hall Assisted STT-RAM Design and Discussion
}

\author{
Enes Eken ${ }^{1}$, Ismail Bayram ${ }^{1}$, Yaojun Zhang ${ }^{1}$, Bonan Yan $^{1}$, Wenqing $\mathrm{Wu}^{2}$, Hai (Helen) $\mathrm{Li}^{1}$, and Yiran $\mathrm{Chen}^{1}$ \\ ${ }^{1}$ Electrical and Computer Engineering, University of Pittsburgh, Pittsburgh, PA \\ ${ }^{2}$ Qualcomm Inc., San Diego, CA
}

\begin{abstract}
In recent years, Spin-Transfer Torque Random Access Memory (STT-RAM) has attracted significant attentions from both industry and academia due to its attractive attributes such as small cell area and non-volatility. However, long switching time and large programming energy of Magnetic Tunneling Junction (MTJ) continue being major challenges in STT-RAM designs. In order to overcome this problem, a Spin-Hall Effect (SHE) assisted STT-RAM structure (SHE-RAM) has been recently invented. In this work, we investigate two possible SHE-RAM designs from the aspects of two different write access operations, namely, High Density SHE-RAM and Disturbance Free SHE-RAM, respectively. In High Density SHE-RAM, SHE current is shared by the entire bit line. Such a structure removes the SHE control transistor from each SHE-RAM cell and hence, substantially reduces the memory cell area. In Disturbance Free SHE-RAM, one memory cell contains two transistors to remove the disturbance to the unselected bits and eliminate the possible erroneous flipping of the bits.
\end{abstract}

Index Terms-SHE-RAM, Spin-Hall Assist, Spintronic.

\section{INTRODUCTION}

Conventional memory technologies like SRAM, DRAM and Flash memory have been widely utilized in modern computer systems. However, as technology node continues to scale down, these electrical charge-based memory technologies suffer from high leakage power and large process variations that cause severe reliability issues. In order to overcome these problems, many new memory technologies, including SpinTransfer Torque Random Access Memory (STT-RAM), have been studied. Although STT-RAM features many attractive characteristics like non-volatility, low standby power, and high cell density [1]-[3], it also has many drawbacks such as long programming latency and high programming energy etc.

Spin-Hall Effect (SHE) assisted STT-RAM (or SHE-RAM) was recently proposed to solve the challenges in conventional STT-RAM designs [4]. By eliminating incubation delay, programming time and/or energy of SHE-RAM cells can be substantially reduced, compared to conventional STT-RAM.

Several access schemes of SHE-RAM were also discussed in [5]. However, these schemes require either very sharp writing pulse or external magnetic field which introduces additional fabrication process/cost. In another design in [6], a single bit is represented by two MTJs and four transistors. This design reduces storage density and also increases access power consumption. Nonetheless, a design that can maximize the benefits of SHE effects is still highly desired.

In this work, we proposed two SHE-RAM designs aiming at different applications. The first one is named as "High

Permission to make digital or hard copies of all or part of this work for personal or classroom use is granted without fee provided that copies are not made or distributed for profit or commercial advantage and that copies bear this notice and the full citation on the first page. Copyrights for components of this work owned by others than ACM must be honored. Abstracting with credit is permitted. To copy otherwise, or republish, to post on servers or to redistribute to lists, requires prior specific permission and/or a fee. Request permissions from permissions@acm.org.

SLIP 16, June 04 2016, Austin, TX, USA

Copyright (C) 2016 ACM. ISBN 978-1-4503-4430-2/16/06. . . \$15.00

DOI: http://dx.doi.org/10.1145/2947357.2947360

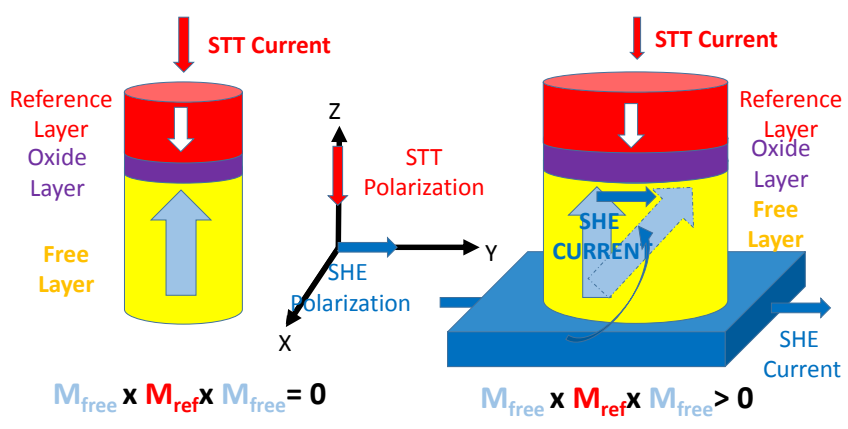

(a)

(b)

Fig. 1. (a) A MTJ whose reference and free layer are in anti-parallel. The exerted torque is zero as the two magnetization vectors are in parallel. (b) Free layer magnetization vector makes an angle under the effect of the SHE current, and STT becomes available to switch the magnetization of the free layer since it is not in parallel to that of the reference layer any more.

Density SHE-RAM", which targets off-chip memory application requiring high cell density. The high cell density of High Density SHE-RAM is ensured by deploying a source line shared by all memory bits to supply the SHE current. Only one transistor is needed to control the whole shared source line. The second one is named as "Disturbance Free SHE-RAM", which targets applications where reliability is the major concern. The potential disturbance to the unselected bits in the High Density SHE-RAM is eliminated in the Disturbance Free SHE-RAM by inserting isolating transistor between the cells and sharing the SHE current among the bits on the same word line.

The remain of this paper is organized as follows: Section II presents the basics of SHE-RAM and the model of spin-hall assist; Section III introduces the designs of High Density SHERAM and Disturbance Free SHE-RAM; Section IV concludes our work.

\section{BASICS OF SHE-RAM}

In conventional STT-RAM, data is stored as the resistance state of a Magnetic Tunneling Junction (MTJ) device, which 
consists of two ferromagnetic layers, namely, reference layer and free layer, and a tunneling oxide layer, as shown in Fig. 1(a). The relative magnetization orientations of these two ferromagnetic layers determine the resistance of the MTJ. That is being said, when their magnetization orientations are in parallel (anti-parallel), the MTJ is in its low (high) resistance state. When a current is injected to the MTJ, the current is spin polarized after passing through the RL, and exerts a torque to the FL and change its orientation.

At the beginning of the switching process of the MTJ, the angle between the magnetization vectors of the free layer and the reference layer will be either $0^{\circ}$ or $180^{\circ}$. In both cases, the torque exerted by the spin polarized current will be zero because the cross product of two vectors with the same direction is zero. In conventional STT-RAM design, this initial angle may be disturbed by thermal fluctuations [4]. The time needed to disturb the free layer magnetization from the "perfect" alignment with the reference layer, called "incubation delay", may be up to several nanoseconds.

Different from conventional STT-RAM, SHE-RAM contains an electrode (e.g. Tantalum) underneath the perpendicular MTJs. Here the magnetization orientation of the two ferromagnetic layers of the MTJ are along the axis $\hat{\mathbf{z}}$. When an assist SHE current pulse is applied on this electrode, an inplane polarized current whose polarization direction is along the axis $\hat{\mathbf{y}}$ is injected into the MTJ. The SHE current pulls the free layers magnetic vector from $0^{\circ}$ or $180^{\circ}$ to an intermediate angle, as shown in Fig. 1(b). Since the magnetization orientations of the free layer and the reference layer are no longer in parallel, the torque exerted by the spin polarized current will be larger than zero. Hence, the SHE current is able to assist the spin polarized current to switch the MTJ much faster. The incubation delay is eliminated and hence, both the switching time and the switching energy consumption are reduced compared to the conventional STT-RAM.

The magnetization dynamics of the MTJ free layer in SHERAM design can be modeled by solving the Landau-LifshitzGilber (LLG) equation [7] with SHE current modification [4] as:

$$
\begin{aligned}
& \frac{\partial \mathbf{M}}{\partial t}=-\gamma \mu_{0}\left(\mathbf{M} \times \mathbf{H}_{\mathrm{eff}}\right)+\frac{\alpha}{M_{s}}\left(\mathbf{M} \times \frac{\partial \mathbf{M}}{\partial t}\right)+ \\
& \frac{c_{\mathrm{SHE}}}{M_{s}^{2}}\left(\mathbf{M} \times \hat{\sigma}_{\mathrm{SHE}} \times \mathbf{M}\right)+\frac{c_{\mathrm{STT}}}{M_{s}^{2}}\left(\mathbf{M} \times \hat{\mathbf{m}}_{\mathrm{ref}} \times \mathbf{M}\right)+ \\
& \frac{\beta_{\mathrm{MTJ}}}{M_{\mathrm{S}}}\left(\mathbf{M} \times \hat{\mathbf{m}}_{\mathrm{ref}}\right),
\end{aligned}
$$

where $\mathbf{M}$ is the magnetization vector of the free layer, $\mathbf{H}_{\mathrm{eff}}$ is the effective magnetic field vector, $\alpha$ is Gilbert damping constant and $M s$ is magnetization saturation, $c_{\mathrm{SHE}}$ is spin-Hall torque coefficient, $c_{\mathrm{STT}}$ is spin-transfer torque coefficient and $\beta_{\mathrm{MTJ}}=0.25 c_{\mathrm{STT}}$ as observed experimentally [4], $\gamma$ is electron gyro magnetic ratio, $\mu_{o}$ is permeability.

\section{SHE-RAM DESIGNS}

In conventional STT-RAM, the MTJ switching can be accelerated by three means: 1) increasing the program current; 2) relaxing the MTJ non-volatility (e.g., by reducing the volume of free layer); and 3) applying an external magnetic field [8]. However, increasing the program current and applying an external magnetic field incur a large power consumption while non-volatility relaxation degrades the retention time of the
TABLE I

SUMMARY OF DEVICE PARAMETERS

\begin{tabular}{|l|l|l|l|}
\hline Device & Parameters & Value & Std. Dev. \\
\hline \multirow{3}{*}{$\begin{array}{l}\text { Tran- } \\
\text { sistor }\end{array}$} & Channel length L & $45 \mathrm{~nm}$ & $2.25 \mathrm{~nm}$ \\
\cline { 2 - 4 } & Channel width W & $90-720 \mathrm{~nm}$ & $2.25 \mathrm{~nm}$ \\
\cline { 2 - 3 } & Threshold voltage $V_{t h}$ & $0.466 \mathrm{~V}$ & $30 \mathrm{mV}$ \\
\hline \multirow{4}{*}{ MTJ } & MTJ Volueme & $45 \times 90 \times 1 \mathrm{~nm}^{3}$ & $5 \%$ \\
\cline { 2 - 3 } & High and low resistance & $2000 / 1000 \Omega$ & \\
\cline { 2 - 3 } & Magnetization saturation & $800 \mathrm{emu} / \mathrm{cc}$ & \\
\cline { 2 - 3 } & Uniaxial anisotropy Hk & $34000 \mathrm{e}$ & \\
\cline { 2 - 4 } & Gilbert damping constant $\alpha$ & 0.01 & \\
\hline
\end{tabular}

STT-RAM cell [9]. As a comparison, spin-hall effect offers a very affordable option for programming performance improvement of the MTJ. Based on spin-hall effect, we propose two SHE-RAM designs, namely, High Density SHE-RAM and Disturbance Free SHE-RAM, aiming different applications. The device parameters used in the relevant analysis are summarized in Table I.

\section{A. High Density SHE-RAM}

High Density SHE-RAM is designed for capacity demanding applications such as off-chip memory. A High Density SHE-RAM cell contains only one transistor and one MTJ, as shown in Fig. 2. A SHE metal wire and a SHE control transistor are shared by all the SHE memory cells connected to the same source line. The programming current to the MTJ of each memory cell is supplied by the transistor connected to the MTJ. During the write operation of a memory cell, both the SHE control transistor and the corresponding cell transistor are turned on simultaneously and the polarization of the programming current is determined by the biases applied on the bit line and the source line.

As a perpendicular MTJ is applied, write operations do not have any requirement on the direction of the SHE current. For example, writing ' 1 ' and ' 0 ' almost equally benefit from a SHE current flowing from SHE control transistor to source line and vice versa becayse SHE currents with both polarizations can disturb the initial alignment of the magnetization direction of the free layer. Similar to the conventional STT-RAM, when the MTJ programming current flows from the bit (source) line to the source (bit) line, the magnetization orientation of the free layer will switch to the same as (opposite to) that of the reference layer, indicating logic ' 0 ' (' 1 ').

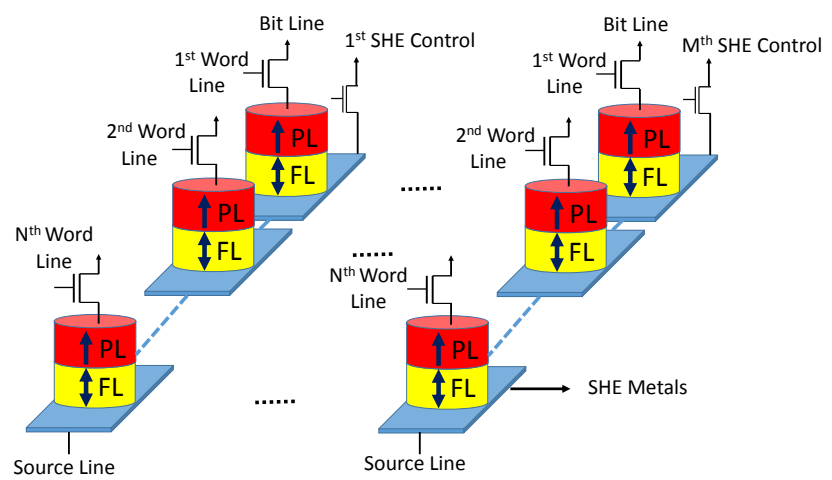

Fig. 2. Illustration of High Density SHE-RAM. SHE current is controlled by the SHE control transistor and MTJs are accessed through the connected word line transistors. During write operations, the data is still written by applying appropriate bias on the source line and the bit line. 


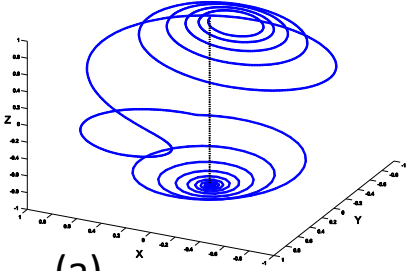

(a)

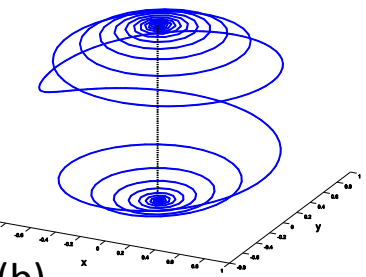

(b)

Fig. 3. (a) Free layer's magnetic moment under the effect of SHE and STT currents. The oscillations starts from the deviated alignment of the free layer. (b) Free layer's magnetic moment under the effect of only STT current. Incubation delay takes place.

The influence of the SHE current on the dynamics of the magnetization vector of the free layer is virtually depicted in Fig. 3(a). When the SHE current is applied, the magnetization vector of the free layer takes almost no time to deviate from the initial position. After that, the magnetization vector of the free layer quickly switches to the target state under the impact of the STT current. As a comparison, if only STT current is applied, significant oscillations occur when the MTJ deviates from the initial position, as shown in Fig. 3(b).

Note that the SHE current is required only at the beginning of the write operation to disturb the initial alignment of the free layer. Hence, for energy saving purpose, the SHE current can be supplied for only a very short time, i.e., sub-nanosecond, rather than the whole writing process. Fig. 4 compares the switching time of the MTJ when different SHE current pulse width is applied under different sizes of the cell transistor in a High Density SHE-RAM cell. The device and circuit level parameters are summarized in TABLE I. The SHE current is fixed at $20 \mu \mathrm{A}$. In general, the longer the SHE current pulse width is, the shorter the MTJ switching time will be: when the transistor width equals $90 \mathrm{~nm}$, raising the SHE current pulse width from $0.5 \mathrm{~ns}$ to $0.7 \mathrm{~ns}$ will reduce the MTJ switching time by half. However, as the transistor width increases, the difference between the switching times of the MTJ at different SHE current pulse widths shrinks, implying the relatively increased impact of the STT current. For comparison purpose, we also simulated the switching time of the MTJ when only STT current is applied. When the transistor width is small,

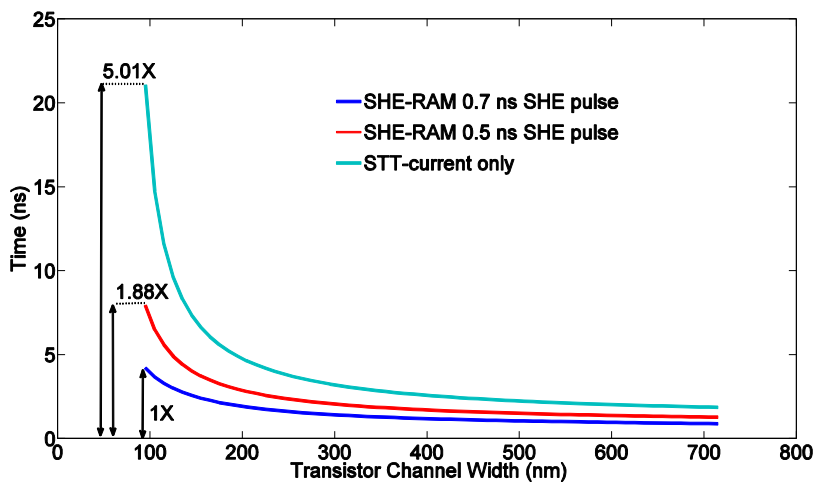

Fig. 4. Simulated MTJ switching time under different transistor widths. Applying SHE current will significantly improve the MTJ switching performance, especially when the transistor width is small (or the STT current is small).

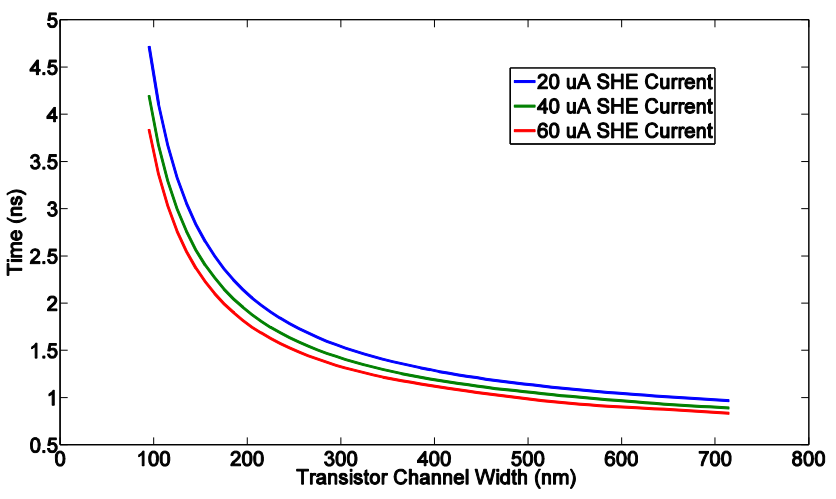

Fig. 5. MTJ switching time of a High Density SHE-RAM cell under different SHE current amplitudes for a $0.7 \mathrm{~ns}$ pulse width.

say, $90 \mathrm{~nm}$, applying a $0.7 \mathrm{~ns}$ SHE current pulse can reduce the MTJ switching time by almost $5 \times$ compared to the case where only the STT current is applied. Again, the MTJ switching time reduction incurred by the SHE current becomes less significant when the transistor width increases. Fig. 5 shows that increasing the amplitude of the SHE current will slightly improve the MTJ switching performance. But this effect is very limited.

The read operation of the High Density SHE-RAM is similar to the conventional STT-RAM: The word line is asserted and a read current $I_{r}$ is injected. Depending on the data stored on the MTJ device (or its resistance state), a high or a low voltage will be generated on the bit line. The value of the stored data can be read out by comparing the bit line voltage with a predefined reference voltage.

We note that the SHE current shared by the memory cells connected to the same source line may cause the disturbance to the unselected cells during write operations when thermal fluctuation is taken into account. Such a disturbance can be further aggravated by the process variations of the MTJ, which result in the variability of the MTJ geometry size, the MTJ critical switching current, and the non-volatility. For example, a $20 \mu \mathrm{A}$ SHE current will result in a disturbance rate of $0.072 \%$ for the unselected MTJ at $300 \mathrm{~K}$. In order to prevent disturbing the unselected bits, we proposed Disturbance Free SHE-RAM.

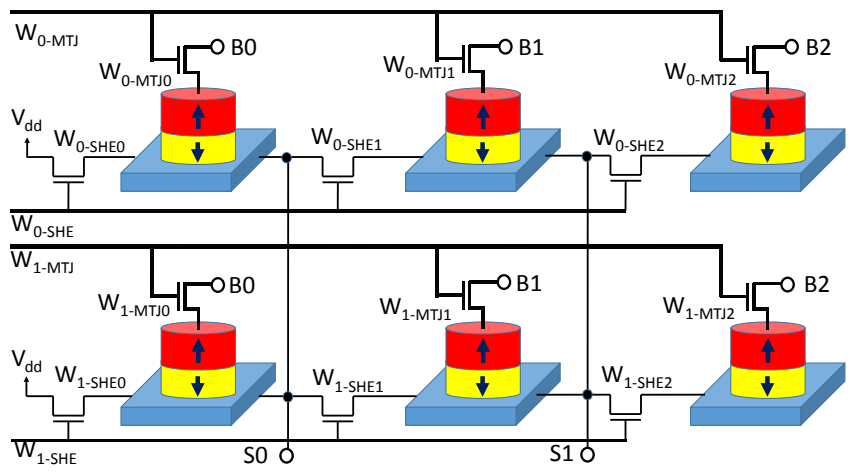

Fig. 6. Disturbance Free SHE-RAM Design. SHE current is shared by the cells on the entire word line and controlled by $\mathrm{W}_{\mathrm{x}-\mathrm{SHE}}$ transistors. 


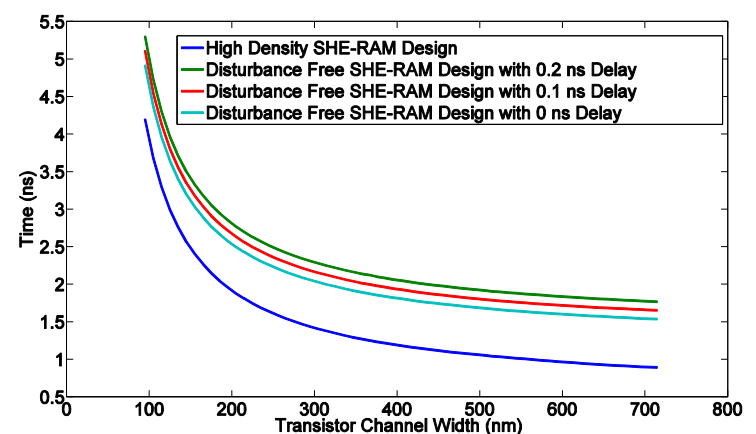

Fig. 7. Switching time comparison for High Density SHE-RAM Design and Disturbance Free SHE-RAM Design for different transistor widths.

\section{B. Disturbance Free SHE-RAM}

Instead of sharing the SHE current among the cells on the same source line in High Density SHE-RAM, in Disturbance Free SHE-RAM, the SHE current is shared among the cells on the same word line, as illustrated in Fig. 6: One Disturbance Free SHE-RAM cell includes two transistors. One of the transistors connects the MTJ and the word line (e.g., $\left.\mathrm{W}_{0-\text { MTJ0 }}\right)$ and the other one is inserted between the segments of the SHE line (e.g., $\left.\mathrm{W}_{0-\mathrm{SHE} 0}\right)$ ). During write operations, the SHE current only passes through the cells that are selected by signal $\mathrm{W}_{\mathrm{x}-\mathrm{SHE}}(\mathrm{x}=0,1, \ldots$ as the word line number $)$. No disturbances to the unselected cells are introduced.

The write operations can be performed as follows: First, SHE line (e.g., $\mathrm{W}_{0-\mathrm{SHE}}$ ) is activated for a duration of subnanosecond to allow the SHE current to flow underneath all the cells along the entire SHE line and disturb their free layer magnetization alignment. After the SHE line is deactivated, MTJ line $\left(\mathrm{W}_{0-\mathrm{MTJ}}\right)$ is turned on to select the cells along the whole MTJ line and the data are programmed into the cells by applying appropriate biases on the bit line and the source line. Since the SHE line has been deactivated before the MTJ line is activated, each cell can be written independently without causing any inference between them. However, the interval between turning on MTJ line and turning off SHE line may adversely affect the SHE effect, as depicted in Fig. 7. Keeping the interval short (but no-zero) is critical for improving the write performance of the Disturbance Free SHE-RAM.

We note that compared to High Density SHE-RAM, Disturbance Free SHE-RAM generally has a longer write operation because the SHE and STT currents are applied at different times, as also shown in Fig. 7. After the SHE line is deactivated, the SHE effect immediately starts to decay. As depicted in Fig. 7, when the interval between turning off the SHE current and turning on the STT current is increasing, the MTJ switching time increases. Nonetheless, Disturbance Free SHE-RAM still demonstrates significantly enhanced write performance compared to conventional STTRAM. Fig. 8 further shows that Disturbance Free SHE-RAM offers not only a faster MTJ switching time but also a tighter distribution of the MTJ switching time when both process variations and thermal fluctuations are considered. This fact holds true over a very wide transistor width range, say, from $90 \mathrm{~nm}$ to $720 \mathrm{~nm}$.

The read operation of Disturbance Free SHE-RAM is also similar to conventional STT-RAM except that transistors

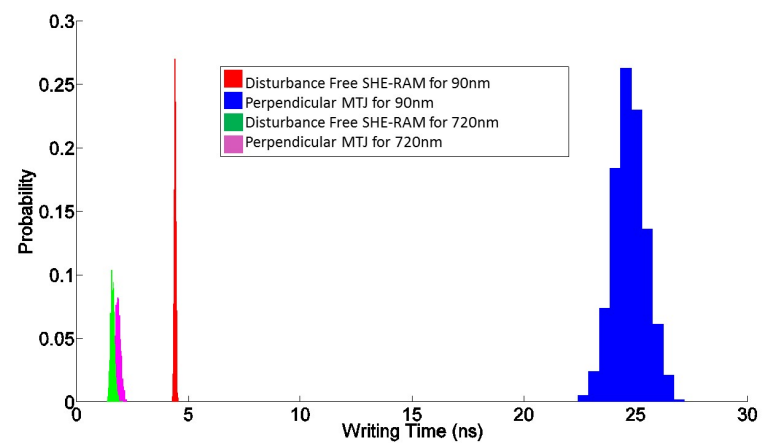

Fig. 8. Switching time probability distribution for Disturbance Free SHERAM Design and for conventional Perpendicular MTJ for $90 \mathrm{~nm}$ and $720 \mathrm{~nm}$ transistor width.

$\mathrm{W}_{\mathrm{x}-\mathrm{SHE}}$ must be turned off in order to operate on each cell independently.

The estimated memory cell areas of High Density SHERAM and Disturbance Free SHE-RAM are $0.0243 \mu \mathrm{m}^{2}$ and $0.0567 \mu \mathrm{m}^{2}$, respectively, at $45 \mathrm{~nm}$ technology node.

\section{CONCLUSION}

In this work, we proposed two designs of SHE-RAM, namely, High Density SHE-RAM and Disturbance Free SHERAM, for capacity-sensitive and reliable applications, respectively. The introduction of SHE current reduces the amplitude of the required switching current to the MTJ, leading to small cell area of High Density SHE-RAM. The disturbance to the unselected cells in High Density SHE-RAM can be then eliminated by the shared word line design in Disturbance Free SHE-RAM though extra cell area overhead is needed.

\section{ACKNOWLEDGMent}

This work was supported in part by NSF CNS-1253424 and AFRL FA8750-15-2-0048.

\section{REFERENCES}

[1] G. Sun, X. Dong, Y. Xie, J. Li, and Y. Chen, "A novel architecture of the $3 \mathrm{~d}$ stacked mram 12 cache for cmps," in High Performance Computer Architecture, 2009. HPCA 2009. IEEE 15th International Symposium on. IEEE, 2009, pp. 239-249.

[2] W. Xu, H. Sun, X. Wang, Y. Chen, and T. Zhang, "Design of last-level on-chip cache using spin-torque transfer ram (stt ram)," Very Large Scale Integration (VLSI) Systems, IEEE Transactions on, vol. 19, no. 3, pp. 483-493, 2011.

[3] P. Zhou, B. Zhao, J. Yang, and Y. Zhang, "Energy reduction for stt-ram using early write termination," in Computer-Aided Design-Digest of Technical Papers, 2009. ICCAD 2009. IEEE/ACM International Conference on. IEEE, 2009, pp. 264-268.

[4] A. van den Brink, S. Cosemans, S. Cornelissen, M. Manfrini, A. Vaysset, W. Van Roy, T. Min, H. Swagten, and B. Koopmans, "Spin-hall-assisted magnetic random access memory," Applied Physics Letters, vol. 104, no. 1, p. 012403, 2014.

[5] K. Jabeur, L. Buda-Prejbeanu, G. Prenat, and G. Pendina, "Study of two writing schemes for a magnetic tunnel junction based on spin orbit torque,' International Journal of Electronics Science and Engineering, vol. 7, no. 8, pp. 501-507, 2013.

[6] Y. Kim, S. H. Choday, and K. Roy, "Dstt-mram: Differential spin hall mram for on-chip memories," arXiv preprint arXiv:1305.4085, 2013.

[7] J. Sun, "Spin-current interaction with a monodomain magnetic body: A model study," Physical Review B, vol. 62, no. 1, p. 570, 2000.

[8] E. Eken, Y. Zhang, W. Wen, R. Joshi, H. Li, and Y. Chen, "A new field-assisted access scheme of stt-ram with self-reference capability," in Design Automation Conference (DAC), 2014 51st ACM/EDAC/IEEE. IEEE, 2014, pp. 1-6.

[9] W. Xu, Y. Chen, X. Wang, and T. Zhang, "Improving stt mram storage density through smaller-than-worst-case transistor sizing," in Proceedings of the 46th Annual Design Automation Conference. ACM, 2009, pp. 8790 . 